\title{
Multichannel Active Noise Control Algorithms Using Inverse Filters
}

M. Bouchard and Yu F.

School of Information Technology and Engineering, University of Ottawa, Ottawa, Ontario, K1N 6N5

\subsection{Introduction}

For active noise control (ANC) systems, a common approach is to use adaptive FIR filters trained with the filtered-x LMS algorithm [1], for both feedforward systems and Internal Model Control (IMC) feedback systems, in monochannel or multichannel systems. Variations of the algorithm sometimes called the modified filtered-x LMS algorithm have been published [2], which can achieve a faster convergence speed by using a larger step size in the algorithm. Fast exact realizations of the filtered- $x$ LMS and the modified filtered-x LMS algorithms have also been published [2]. In most cases, these fast realizations can reduce the computational complexity of the filtered-x LMS and modified filtered-x LMS algorithms for multichannel systems.

Many algorithms that can achieve a faster convergence than the multichannel filtered- $x$ LMS or the modified filtered-x LMS algorithms for ANC systems have also been published over the years. However, these algorithms can only provide increased convergence speed at the cost of increased computational load, compared to the multichannel filtered-x LMS algorithm or its fast exact realizations. In this paper, a simple multichannel algorithm that can both reduce the computational load and increase the convergence speed compared to the multichannel filtered-x LMS algorithm or its fast exact realizations is introduced, using an inverse structure and filtered-x LMS-based algorithms.

In the proposed approach, the standard FIR controller is split into two parts: multichannel predictors and multichannel delayed noncausal models of the inverse plant between some error sensors (typically microphones in ANC systems) and some actuators (typically loudspeakers). Figure 1 shows the structure of the proposed approach for a simplified monochannel case, using the delayed non-causal filter.

With the proposed inverse structure that uses adaptive FIR filters, there are two benefits of using delayed non-causal filters modeling the inverse plant of an ANC system. One benefit is that the combination of the delayed non-causal models and the models of the direct plant becomes approximately pure delays (approximately because models are never perfect). Using these pure delay operations can eliminate some costly convolutions. The second benefit of using the delayed non-causal filters is due to the fact that the convergence speed of filtered-x LMS-based algorithms is related to the eigenvalue spread in the correlation matrix of the filtered reference signals in the algorithms. This eigenvalue spread can be typically reduced using the proposed structure, because the resulting combination of the delayed noncausal models and the models of the direct plant will have flat, uniform frequency responses, thus eliminating the eigenvalue spread caused by filtering reference signals with the models of the direct plant in the standard filtered-x LMS structure [1].

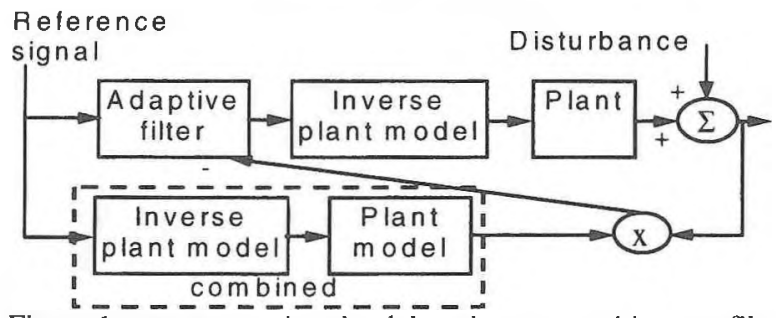

Figure $\overline{1}$ : structure using the delayed non-causal inverse filter

\subsection{Description of the inverse algorithms}

To describe explicitly the proposed the multichannel inverse filtered-x LMS (IFX) and inverse modified filtered-x LMS (IMFX) algorithms, the following notation is defined:

$I, J, K \quad$ number of reference sensors, actuators and error sensors in a feedforward ANC system

$e_{k}(n) \quad$ value at time $n$ of the signal measured by the $k^{\text {th }}$ error sensor

$x_{i}(n) \quad$ value at time $n$ of the $i^{\text {th }}$ reference signal in a feedforward ANC system (measured with a reference sensor)

$t_{k}(n) \quad$ estimated value of the disturbance signal $d_{k}(n+D)$ at time $n$, where $D$ is the delay required to make the FIR filter modeling the inverse plant causal. $t_{k}(n)$ is obtained by filtering the reference signals $x_{i}(n)$ with the adaptive FIR filters.

$y_{j}(n) \quad$ value at time $n$ of the $j^{\text {th }}$ actuator signal

$z_{i, j, k}(n)$ value at time $n$ of the signal obtained by filtering the reference signal $x_{i}(n)$ with the FIR filter modeling the inverse plant between $e_{k}(n)$ and $y_{j}(n)$

$v_{i, k^{\prime}, k}(n)$ value at time $n$ of the filtered reference signal, i.e. the signal obtained by filtering the $z_{i, j, k^{\prime}}(n)$ signal with the FIR filter modeling the direct plant between $y_{j}(n)$ and $e_{k}(n)$

$d_{k}^{\prime}(n) \quad$ value at time $n$ of the estimated primary sound field at the $k^{\text {th }}$ error sensor (for the modified structure only [2])

$e_{k}^{\prime}(n) \quad$ value at time $n$ of the alternative error computed in the modified structure [2]

$w_{i, k, l}(n)$ value at time $n$ of the $l^{\text {th }}$ coefficient in the adaptive FIR filter linking $x_{i}(n)$ and $t_{k}(n)$

$h_{j, k, m}$ value of the $m^{\text {th }}$ coefficient in the non-adaptive FIR filter model of the direct plant between $y_{j}(n)$ and $e_{k}(n)$.

$g j, k, p$ value of the $p^{\text {th }}$ coefficient in the non-adaptive FIR filter modeling the inverse plant between $e_{k}(n)$ and $y_{j}(n)$.

$h_{j, k}, g_{j, k}, w_{i, k}(n)$ column vectors containing the $h_{j, k, m}$. $g_{j, k, p}$, and $w_{i, k, l}(n)$ coefficients, respectively $x_{i}(n), t_{k}(n), y_{j}(n), z_{i, j, k}(n), v_{i, k^{\prime}, k}(n)$ column vectors containing the current and previous values of $x_{i}(n)$, $t_{k}(n), y_{j}(n), z_{i, j, k}(n)$ and $v_{i, k^{\prime}, k}(n)$, respectively.

The inverse filtered-x LMS (IFX) algorithm is first described, using equations (1)-(5). In these equations, $k$ and $k^{\prime}$ are both used as an index for the error sensor signals or their prediction:

$$
t_{k}(n)=\sum_{i=1}^{I} w_{i, k}(n)^{T} x_{i}(n)
$$




$$
\begin{aligned}
& y_{j}(n)=\sum_{k=1}^{K} g_{j, k} T_{t_{k}(n)} \\
& z_{i, j, k}(n)=g_{j, k} T_{x_{i}^{\prime}(n)}^{J} \\
& v_{i, k^{\prime}, k}(n)=\sum_{j=1}^{J} h_{j, k^{\prime}} z_{i, j, k^{\prime}}(n) \\
& w_{i, k^{\prime}(n+1)}=w_{i, k^{\prime}}(n)-\mu \sum_{k=1}^{K} v_{i, k^{\prime}, k}(n) e_{k}(n)
\end{aligned}
$$

where $\mu$ is a scalar convergence gain. Note that the $g_{j, k}$ filters in (3) can be convoluted with the $\boldsymbol{h}_{j, k}$ filters in (4), and these convolutions only need to be computed once. Therefore, equations (3).(4) can be combined in a single equation, which will significantly reduce the number of computations of the algorithm:

$v_{i, k^{\prime}, k}(n)=\left(\sum_{j=1}^{J} g_{j, k^{\prime}} * \boldsymbol{h}_{j, k}\right)^{T} \boldsymbol{x}_{i}(n)$

where "* " is the convolution operator and $\sum_{j=1}^{J} g_{j, k}{ }^{*} \boldsymbol{h}_{j, k}$ is only computed once, offline. Since $\boldsymbol{h}_{j, k}$ and $\boldsymbol{g}_{j, k}$ are models of the direct and inverse plant, their combination can also be estimated by pure delays (the same delay required to make $g$ j,k causal). Therefore, it is possible to further simplify equations (3)-(5) and use (7) instead, to have a simplified IFX algorithm:

$w_{i, k}(n+1)=w_{i, k}(n)-\mu x_{i}(n-D) e_{k}(n)$

where $D$ is the delay required to make $\boldsymbol{g} j, k$ causal.

The inverse modified filtered-x LMS (IMFX) algorithm is described next. It combines the modified filtered-x LMS algorithm [2] with the inverse structure that uses the models of the inverse plant. The algorithm can be described by equations (1),(2),(6) and $(8)-(10)$ :

$$
\begin{aligned}
& d_{k}^{\prime}(n)=e_{k}(n)-\sum_{j=1}^{J} h_{j, k^{\prime}}{ }^{T} y_{j}(n) \\
& e_{k}^{\prime}(n)=d_{k}^{\prime}(n)+\sum_{i=1 k^{\prime}=1}^{l} \sum_{i, k^{\prime}(n)}^{K} w_{v_{i, k^{\prime}, k}(n)} \\
& \boldsymbol{w}_{i, k^{\prime}}(n+1)=w_{i, k^{\prime}}(n)-\mu \sum_{k=1}^{K} v_{i, k^{\prime}, k}(n) e_{k}^{\prime}(n)
\end{aligned}
$$

Again, since the combination of $h_{j, k}$ and $\boldsymbol{g}_{j, k}$ results in pure delays, a simplified IMFX algorithm can be described by equations (1),(2) and by (11)-(13):

$$
\begin{aligned}
& d_{k}^{\prime}(n)=e_{k}(n)-t_{k}(n-D) \\
& e_{k}^{\prime}(n)=d_{k}^{\prime}(n)+\sum_{i=1}^{I} w_{i, k}(n)^{T} x_{i}(n-D) \\
& w_{i, k}(n+1)=w_{i, k}(n)-\mu x_{i}(n-D) e_{k}^{\prime}(n)
\end{aligned}
$$

\subsection{Computational load of the algorithms}

The computational load of the simplified IFX and simplified IMFX algorithms was compared with the computational load of the fast exact versions of the filtered-x LMS and the modified filtered-x LMS found in [2]. Detailed results can be found in [3],[4]. For a system with $I=1, J=4, K=4,300$ coefficients for the adaptive filters, 256 coefficients for the direct plant models and 2.56 coefficients for the inverse plant models, the fast exact realizations of the standard filtered-x LMS and modified filtered-x algorithm require 8030 and 32606 multiplies per iteration. respectively. For the same system, the simplified IFX and IMFX algorithms require only 6100 and 7100 multiplies per iteration, respectively. There is thus a potential for a significant reduction of the computational load using the proposed inverse algorithm, in particular for algorithms using the modified structure [2].

\subsection{Simulation results}

To evaluate the potential gain in convergence speed, simulations were performed using an acoustic plant measured on a headphone. The plant and its inverse are described in [3].[4]. The inverse impulse response of the plant required a delay of 15 samples to become approximately causal [3],[4]. The convergence speed of the filtered-x LMS algorithm, the modified filtered-x LMS algorithm, the IFX/IMFX algorithms and their simplified versions appears in Fig. 2, for a $20 \mathrm{~dB}$ SNR on the plant direct and inverse models. All the algorithms using the inverse filter produced a better convergence performance, as expected, and the simplification using a pure delay did not affect much the performance of the inverse algorithms for this system. With a SNR of more than $10 \mathrm{~dB}$ on the plant direct and inverse models, the algorithms using the inverse filter produced a faster convergence speed. However in the SNR was less or equal to $10 \mathrm{~dB}$, then the classical algorithms performed better.

\subsection{Conclusion}

In this paper, an inverse structure was introduced for the use of adaptive FIR filters in ANC systems. Multichannel adaptive FIR filter learning algorithms based on the filtered-x LMS algorithm were introduced for this inverse structure. It was shown with simulations using a realistic acoustical plant that some versions of the introduced algorithms can achieve both a reduction of the computational load and an increase of the convergence speed. compared to standard algorithms for ANC such as the multichannel filtered-x LMS algorithm, the modified filtered-x LMS algorithm or their fast exact realizations. Some theoretical work on the effect of plant model and inverse plant model errors would be of interest.

\section{References}

[1] S.M. Kuo and D.R. Morgan, Active noise control systems : algorithms and DSP implementations, New-York: J. Wiley \& Sons, 1996

[2] S. Douglas, "Fast, exact filtered-x LMS and LMS algorithms for multichannel active noise control", Proc. ICASSP-97, Munich. Germany, 1997, pp.399-402

[3] Bouchard, M., and $\mathrm{Yu}, \mathrm{F}$. Inverse structure for active noise control and combined active noise control/sound reproduction systems, accepted for publication in IEEE Trans. on Speech and Audio Processing (scheduled for September 2000)

[4] $\mathrm{Yu}, \mathrm{F}$. and Bouchard, M. Multichannel active noise control algorithms using inverse filters, ICASSP 2000, Vol. II, 825-828 (Istanbul, Turkey, June 2000)

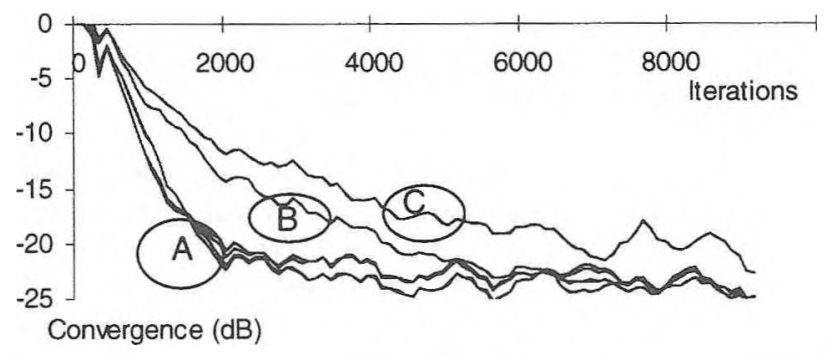

Figure 2: convergence curves of A) IFX/IMFX algorithms B) modified filtered-x LMS algorithm $C$ ) filtered-x LMS algorithm 